\title{
An Efficient High Order Well-Balanced Finite Difference WENO Scheme for the Blood Flow Model
}

\author{
Shouguo Qian ${ }^{1,2}$, Gang $\mathrm{Li}^{2}$, Xianqing $\mathrm{Lv}^{3}$ and Fengjing Shao ${ }^{1, *}$ \\ ${ }^{1}$ Institute of Complexity Science, Qingdao University, Qingdao, Shandong 266071, \\ China \\ ${ }^{2}$ School of Mathematics and Statistics, Qingdao University, Qingdao, Shandong 266071, \\ China \\ ${ }^{3}$ Laboratory of Physical Oceanography, Ocean University of China, Qingdao, \\ Shandong 266100, China
}

Received 15 October 2016; Accepted (in revised version) 13 April 2017

AMS subject classifications: 65L12,74S20

\begin{abstract}
The blood flow model admits the steady state, in which the flux gradient is non-zero and is exactly balanced by the source term. In this paper, we present a high order well-balanced finite difference weighted essentially non-oscillatory (WENO) scheme, which exactly preserves the steady state. In order to maintain the wellbalanced property, we propose to reformulate the equation and apply a novel source term approximation. Extensive numerical experiments are carried out to verify the performances of the current scheme such as the maintenance of well-balanced property, the ability to capture the perturbations of such steady state and the genuine high order accuracy for smooth solutions.
\end{abstract}

Key words: Blood flow model, finite difference scheme, WENO scheme, high order accuracy, well-balanced property.

\section{Introduction}

In this paper, we are interested in numerical computing the blood flow model in arteries by high order schemes. Numerical simulations with high order accuracy for the blood flow model have wide applications in medical engineering [1,2]. In one spatial dimension, the blood flow model takes the following system of hyperbolic balanced laws [3]:

$$
\left\{\begin{array}{l}
A_{t}+Q_{x}=0 \\
Q_{t}+\left(\frac{Q^{2}}{A}+\frac{K}{3 \rho \sqrt{\pi}} A^{\frac{3}{2}}\right)_{x}=\frac{K A}{2 \rho \sqrt{\pi} \sqrt{A_{0}}}\left(A_{0}\right)_{x}
\end{array}\right.
$$

\footnotetext{
*Corresponding author.

Emails: qianshouguo@aliyun.com (S. G. Qian), gangli1978@163.com (G. Li), xqinglv@ouc.edu.cn (X. Q. Lv), sfj@qdu.edu.cn (F. J. Shao)
} 
where $A=\pi R^{2}$ is the cross-sectional area with $R$ being the radius of the vessel, $Q=A u$ denotes the discharge, $u$ means the flow velocity, $\rho$ stands for the blood density and $K$ represents the arterial stiffness. In addition, $A_{0}=\pi R_{0}^{2}$ is the cross section at rest $(u=0 \mathrm{~m} / \mathrm{s})$ with $R_{0}$ being the radius of the vessel, which may be variable in the case of aneurism, stenosis or taper.

The important property of the model (1.1) is that it admits the steady state, also called mechanical equilibrium, where the flux gradient is exactly balanced by the source term:

$$
u=0 \text { and } A=A_{0} .
$$

Under the above steady state (1.2), the flux gradient is non-zero and is exactly balanced by the source term. Consequently, it is desirable to maintain the balancing between the flux gradient and the source term at the discrete level. In general, the standard numerical schemes generally fail to satisfy the discrete version of this balance exactly at the steady state, even introduce spurious oscillations, unless the mesh size is extremely refined. However, the mesh refinement procedure is not applicable for practical problems due to the very high computational cost. In 1996, Greenberg et al. [4] originally introduced the well-balanced schemes, which can preserve exactly the steady state solutions up to machine accuracy at the discrete level. Moreover, well-balanced schemes can capture small perturbations well even on relatively coarse meshes [5]. It is important to note that many attempts have been concentrated on the well-balanced schemes for the shallow water equations using different approaches, see among others [6-11] and references therein.

In recent years, there have been many interesting attempts on the well-balanced schemes for the blood flow model. For example, Delestre and Lagrée [12] presented a well-balanced finite volume scheme based on the conservative governing equations [13-15]. Müller et al. [16] constructed a well-balanced high order finite volume scheme for the blood flow in elastic vessels with varying mechanical properties. Recently, Murillo et al. [17] have presented an energy-balanced approximate solver with upwind discretization for the source term. More recently, Wang et al. [18] have presented a well-balanced finite difference WENO scheme based on the splitting algorithm of the source term.

The key objective of this research is to develop an efficient high order well-balanced finite difference WENO scheme based on a reformation of the source term to avoid the splitting of the source term as in [18]. Rigorous numerical analysis as well as extensive numerical experiments all verify the satisfaction of the well-balanced property of the resulting scheme. In order to obtain well-balanced finite difference WENO schemes, we firstly reformulate the source term in an equivalent form, then construct linear finite $\mathrm{d}$ ifference operator coupled with modification of the flux splitting. The above procedures lead to an efficient WENO scheme compared with the WENO scheme in [18]. In addition, this WENO scheme keeps high order accuracy for smooth solutions, and enjoys steep discontinuity transition at the same time.

This paper is organized as follows: we briefly review the key idea of the finite difference WENO schemes in Section 2. In Section 3, we propose a high order well-balanced 
finite difference WENO scheme for the blood flow model. Extensive numerical experiments are carried out in Section 4. Some conclusions are given in Section 5.

\section{A review of high order finite difference WENO schemes}

The first finite difference WENO scheme was introduced in 1996 by Jiang and Shu [19] for hyperbolic conservation laws. More detailed information of WENO schemes can be found in the lecture note [20]. For the latest advances regarding WENO schemes, we refer to the review [21].

For the sake of simplicity, we assume that the grid points $\left\{x_{j}\right\}$ are uniformly distributed with cell size $\Delta x=x_{j+1}-x_{j}$ and we denote the cells by $I_{j}=\left[x_{j-1 / 2}, x_{j+1 / 2}\right]$ with $x_{j+1 / 2}=x_{j}+\Delta x / 2$ being the center of the cell $I_{j}$.

Firstly, we take the one-dimensional (1D) scalar hyperbolic conservation laws

$$
u_{t}+f(u)_{x}=0
$$

as an example to illustrate the finite difference WENO schemes. For conservative schemes, we apply the conservative flux difference to approximate the flux gradient $f(u)_{x}$, i.e.,

$$
\left.f(u)_{x}\right|_{x=x_{j}} \approx \frac{1}{\Delta x}\left(\widehat{f}_{j+\frac{1}{2}}-\widehat{f}_{j-\frac{1}{2}}\right) .
$$

Denote the point value $u\left(x_{j}, t\right)$ by $u_{j}(t)$, we obtain the following high order semi-discrete conservative finite difference schemes

$$
\frac{d}{d t} u_{j}(t)=-\frac{1}{\Delta x}\left(\widehat{f}_{j+\frac{1}{2}}-\widehat{f}_{j-\frac{1}{2}}\right),
$$

where $\widehat{f}_{j+\frac{1}{2}}$ is the numerical flux to approximate $h_{j+\frac{1}{2}}=h\left(x_{j+\frac{1}{2}}\right)$ with high order accuracy. Here $h(x)$ is implicitly defined as in [19]

$$
f(u(x))=\frac{1}{\Delta x} \int_{x-\Delta x / 2}^{x+\Delta x / 2} h(\xi) d \xi .
$$

In addition, we take upwinding into account to maintain the numerical stability and split a general flux into two parts

$$
f(u)=f^{+}(u)+f^{-}(u),
$$

where

$$
\frac{d f^{+}(u)}{d u} \geq 0 \quad \text { and } \quad \frac{d f^{-}(u)}{d u} \leq 0 .
$$

Herein, we take the simple Lax-Friedrichs flux

$$
f^{ \pm}(u)=\frac{1}{2}(f(u) \pm \alpha u),
$$


where $\alpha=\max _{u}\left|f^{\prime}(u)\right|$. With respect to whether the maximum is taken globally (along the line of computation) or locally, we call this scheme as the Lax-Friedrichs WENO scheme (denoted by WENO-LF). With respect to $f^{+}(u)$ and $f^{-}(u)$, we can get numerical fluxes $\widehat{f}_{j+\frac{1}{2}}^{+}$and $\widehat{f}_{j+\frac{1}{2}}^{-}$using the WENO reconstruction, respectively. Finally, we get the numerical flux as follows

$$
\widehat{f}_{j+\frac{1}{2}}=\widehat{f}_{j+\frac{1}{2}}^{+}+\widehat{f}_{j+\frac{1}{2}}^{-} .
$$

By means of the WENO reconstruction procedure, $\widehat{f}_{j+\frac{1}{2}}^{+}$is expressed as [19]

$$
\widehat{f}_{j+\frac{1}{2}}^{+}=\sum_{k=0}^{r} \omega_{k} q_{k}^{r}\left(f_{j+k-r}^{+}, \cdots, f_{j+k}^{+}\right)
$$

where $\omega_{k}$ is the nonlinear weight, $f_{i}^{+}=f^{+}\left(u_{i}\right), i=j-r, \cdots, j+r$, and

$$
q_{k}^{r}\left(\mathrm{~g}_{0}, \cdots, \mathrm{g}_{r}\right)=\sum_{l=0}^{r} a_{k, l}^{r} \mathrm{~g}_{l}
$$

is the low order approximation to $\widehat{f}_{j+\frac{1}{2}}^{+}$on the $k$ th stencil $S_{k}=\left(x_{j+k-r}, \cdots, x_{j+k}\right), k=0,1, \cdots, r$, and $a_{k, l}^{r}, 0 \leq k, l \leq r$ are constant coefficients, see [20] for more details.

The nonlinear weights $\omega_{k}$ in (2.4) are designed to yield $(2 r+1)$ th-order accuracy in smooth regions of the solution. In $[19,20]$, the nonlinear weight $\omega_{k}$ is formulated as

$$
\omega_{k}=\frac{\alpha_{k}}{\sum_{l=0}^{r} \alpha_{l}} \quad \text { with } \alpha_{k}=\frac{C_{k}^{r}}{\left(\varepsilon_{\mathrm{WENO}}+I S_{k}\right)^{2}}, \quad k=0,1, \cdots, r,
$$

where $C_{k}^{r}$ is the linear weight, $\varepsilon_{\text {WENO }}$ is a small constant used here to avoid the denominator becoming zero, and $\varepsilon_{\mathrm{WENO}}=10^{-6}$ is used in all test cases in this paper. $I S_{k}$ is a smoothness indicator of $f^{+}(u)$ on stencil $S_{k}, k=0,1, \cdots, r$, and we employ the smoothness indicators proposed in $[19,20]$, i.e.,

$$
I S_{k}=\sum_{l=1}^{r} \int_{x_{j-\frac{1}{2}}}^{x_{j+\frac{1}{2}}}(\Delta x)^{2 l-1}\left(q_{k}^{(l)}\right)^{2} d x,
$$

where $q_{k}^{(l)}$ is the $l$ th-derivative of $q_{k}(x)$ which is the reconstruction polynomial of $f^{+}(u)$ on stencil $S_{k}$ such that

$$
\frac{1}{\Delta x} \int_{I_{i}} q_{k}(x) d x=f_{i}^{+}, \quad i=j+k-r, \cdots, j+k
$$

The WENO reconstruction procedure for $\widehat{f}_{j+\frac{1}{2}}^{-}$is a mirror symmetry to that of $\widehat{f}_{j+\frac{1}{2}}^{+}$ with respect to $x_{j+\frac{1}{2}}$. Consequently, the numerical flux $\widehat{f}_{j+\frac{1}{2}}$ is then calculated by

$$
\widehat{f}_{j+\frac{1}{2}}=\widehat{f}_{j+\frac{1}{2}}^{+}+\widehat{f}_{j+\frac{1}{2}}^{-} .
$$


Ultimately, we obtain the semi-discrete scheme (2.2).

For hyperbolic systems of conservation laws, we usually apply the local characteristic decomposition procedure with more computational cost to obtain good numerical results, which is more robust than a component by component version. The complete algorithm can be found in $[20,21]$.

For the temporal discretization, we apply the third order total variation diminishing (TVD) Runge-Kutta method [22]

$$
\begin{aligned}
& U^{(1)}=U^{n}+\Delta t \mathcal{F}\left(U^{n}\right), \\
& U^{(2)}=\frac{3}{4} U^{n}+\frac{1}{4}\left(U^{(1)}+\Delta t \mathcal{F}\left(U^{(1)}\right)\right), \\
& U^{n+1}=\frac{1}{3} U^{n}+\frac{2}{3}\left(U^{(2)}+\Delta t \mathcal{F}\left(U^{(2)}\right)\right),
\end{aligned}
$$

with $\mathcal{F}(U)$ being the spatial operator.

\section{An efficient WENO scheme for the blood flow model}

In this section, we present a high order well-balanced finite difference WENO scheme for the blood flow model (1.1). The key idea is to discretize the source term by a finite difference WENO formula, which is consistent with that for the flux gradient.

\subsection{Reformulation of the equation}

In order to construct a well-balanced finite difference scheme, the first step is to reformulate the source term $\frac{K A}{2 \rho \sqrt{\pi} \sqrt{A_{0}}}\left(A_{0}\right)_{x}$ in an equivalent form $\frac{K}{3 \rho \sqrt{\pi}} \frac{A}{A_{0}}\left(A_{0}^{\frac{3}{2}}\right)_{x}$ and this step is a key procedure of the current scheme. Therefore the original system (1.1) becomes as follows

$$
\left\{\begin{array}{l}
A_{t}+Q_{x}=0, \\
Q_{t}+\left(\frac{Q^{2}}{A}+\frac{K}{3 \rho \sqrt{\pi}} A^{\frac{3}{2}}\right)_{x}=\frac{K}{3 \rho \sqrt{\pi}} \frac{A}{A_{0}}\left(A_{0}^{\frac{3}{2}}\right)_{x^{\prime}}
\end{array}\right.
$$

which can be denoted in a compact vector form

$$
U_{t}+f(U)_{x}=S\left(A, A_{0}\right),
$$

where $U=(A, Q)^{\top}$ with the superscript $\top$ denoting the transpose, $f(U)$ stands for the flux and $S\left(A, A_{0}\right)$ represents the source term. The main motivation of such a modification in (3.1) is to let the source term and the corresponding flux gradient share similar discretization form in the case of the steady state (1.2). 


\subsection{Novel source term approximation}

Herein, the semi-discrete WENO scheme takes the form of

$$
\frac{d}{d t} U_{j}(t)+\frac{1}{\Delta x_{j}}\left(\widehat{F}_{j+\frac{1}{2}}-\widehat{F}_{j-\frac{1}{2}}\right)=S_{j}
$$

but with slightly modified numerical fluxes and source term approximations outlined below.

To present the basic idea of the modification, we first consider the situation when the WENO scheme is applied without the flux splitting (e.g., WENO-Roe scheme) and the local characteristic decomposition for the system of equations. Let's compute the numerical fluxes $\widehat{F}_{j+\frac{1}{2}}$ as stated in Section 2. For the source term $\frac{K}{3 \rho \sqrt{\pi}} \frac{A}{A_{0}}\left(A_{0}^{\frac{3}{2}}\right)_{x}$, we use the usual WENO reconstruction to compute $\left(\widehat{A_{0}^{\frac{3}{2}}}\right)_{j+\frac{1}{2}}$ from the neighbour point values $\left(A_{0}^{\frac{3}{2}}\right)_{j \pm k}$, and then approximate $\left(\left(A_{0}^{\frac{3}{2}}\right)_{x}\right)_{j}$ as follows

$$
\left(\left(A_{0}^{\frac{3}{2}}\right)_{x}\right)_{j}=\frac{\left.\widehat{\left(A_{0}^{\frac{3}{2}}\right.}\right)_{j+\frac{1}{2}}-\left(\widehat{A_{0}^{\frac{3}{2}}}\right)_{j-\frac{1}{2}}}{\Delta x}
$$

Subsequently, we approximate the source term in the following form:

$$
\left(\frac{K}{3 \rho \sqrt{\pi}} \frac{A}{A_{0}}\left(A_{0}^{\frac{3}{2}}\right)_{x}\right)_{j}=\left(\frac{K}{3 \rho \sqrt{\pi}} \frac{A}{A_{0}}\right)_{j} \frac{\left(\widehat{A_{0}^{\frac{3}{2}}}\right)_{j+\frac{1}{2}}-\left(\widehat{A_{0}^{\frac{3}{2}}}\right)_{j-\frac{1}{2}}}{\Delta x} .
$$

With the standard WENO numerical flux and the source term approximation stated above, this WENO scheme without any further modification is actually well-balanced, and we refer to Proposition 3.1 below for a detailed proof.

Subsequently, we look at the situation when the WENO reconstruction procedure involves the local characteristic decomposition, which is often applied for the system of equations including the Euler equations and shallow water equations for the sake of robustness. The local characteristic matrix $R$, consisting of the right eigenvectors of the Jacobian matrix, is used to compute the numerical flux at point $x_{j+\frac{1}{2}}$. All the neighboring point values of the vectors $\left(Q, \frac{Q^{2}}{A}+\frac{K}{3 \rho \sqrt{\pi}} A^{\frac{3}{2}}\right)_{j \pm k}^{\top}$ are all projected to the local characteristic fields determined by $R^{-1}$, and the WENO reconstruction is then completed in the characteristic space. After the WENO reconstruction, the value will be projected back to the physical space to obtain $\widehat{F}_{j+\frac{1}{2}}$. For the source term approximation, we mimic the process and project the vector $\left(0, A_{0}^{\frac{3}{2}}\right)_{j \pm k}^{\top}$ to the local characteristic fields to carry out the WENO reconstruction. The reconstructed value is also projected back to obtain $\left(\widehat{A_{0}^{\frac{3}{2}}}\right)_{j \pm \frac{1}{2}}$ for the source term approximation. 
Finally, we consider WENO schemes with a Lax-Friedrichs flux splitting, denoted by the WENO-LF scheme. The flux $F(U)$ is separated into two parts:

$$
F(U)=F^{+}(U)+F^{-}(U),
$$

where

$$
F^{ \pm}(U)=\frac{1}{2}\left(F(U) \pm \alpha_{i} U\right)
$$

with

$$
\alpha_{i}=\max _{U}\left|\lambda_{i}(U)\right|
$$

for the $i$-th characteristic field and $\lambda_{i}(U)$ being the $i$-th eigenvalue of the Jacobian matrix $\partial F(U) / \partial U$. The $\pm \alpha_{i} U$ term contributes to the numerical viscosity, which is essential for the numerical approximation of hyperbolic conservation laws. But such term may destroy the well-balanced property at the steady state solution. Therefore, we propose to modify the flux splitting as follows

$$
F^{ \pm}(U)=\frac{1}{2}\left(F(U) \pm \alpha_{i} \widetilde{U}\right)
$$

with $\widetilde{U}$ given by

$$
\widetilde{U}=\left(A-A_{0}, Q\right)^{T} .
$$

At the steady state (1.2), $\widetilde{U}$ becomes the constant, and the effect of this viscosity term $\pm \alpha_{i} \widetilde{U}$ towards the approximation of $F(U)_{x}$ is zero. As the flux splitting WENO approximation becomes $F^{ \pm}(U)=F(U) / 2$ at the steady state, the well-balanced source term approximation can be obtained if we simply split the derivatives in the source term as:

$$
\left(\begin{array}{c}
0 \\
A_{0}^{\frac{3}{2}}
\end{array}\right)_{x}=\frac{1}{2}\left(\begin{array}{c}
0 \\
A_{0}^{\frac{3}{2}}
\end{array}\right)_{x}+\frac{1}{2}\left(\begin{array}{c}
0 \\
A_{0}^{\frac{3}{2}}
\end{array}\right)_{x}
$$

and apply the same WENO reconstruction procedure corresponding to $F^{+}$or $F^{-}$to approximate them respectively. We then add up the resulting $\widehat{\left(A_{0}^{\frac{3}{2}}\right)_{j+\frac{1}{2}}^{ \pm}}$to obtain

$$
\left(\widehat{A_{0}^{\frac{3}{2}}}\right)_{j+\frac{1}{2}}=\left(\widehat{A_{0}^{\frac{3}{2}}}\right)_{j+\frac{1}{2}}^{+}+\left(\widehat{A_{0}^{\frac{3}{2}}}\right)_{j+\frac{1}{2}}^{-} .
$$

Remark 3.1. Herein, we only reformulate the source term in an equivalent form and avoid the splitting of the source term as in [18]. In other words, there is only one derivative in the reformulation of the source term, while there are two derivatives with the splitting of the source term in [18]. So the current finite difference WENO scheme is more efficient than the one in [18]. 


\subsection{Well-balanced scheme}

For the finite difference WENO scheme described above, we have

Proposition 3.1. For the blood flow model (1.1), reformulated in the form of (3.1), the semi-discrete finite difference WENO scheme (3.2), with the standard WENO numerical fluxes for WENO-Roe or modified numerical fluxes based on (3.5) for WENO-LF, combined with the source term approximation stated in (3.3) for WENO-Roe or modified source term approximation with (3.3), (3.6), (3.7) for WENO-LF, are well-balanced for the steady state solution (1.2).

Proof. We take the WENO-Roe scheme as an example to prove the well-balanced property. The proof of WENO-LF method follows the same way and is ignored here to save space. At the steady state (1.2) where $u=0$ and $A=A_{0}$, the flux $F(U)$ becomes

$$
F(U)=\left(\begin{array}{c}
Q \\
\frac{Q^{2}}{A}+\frac{K}{3 \rho \sqrt{\pi}} A^{\frac{3}{2}}
\end{array}\right)=\left(\begin{array}{c}
0 \\
\frac{K}{3 \rho \sqrt{\pi}} A^{\frac{3}{2}}
\end{array}\right),
$$

and the numerical flux $\widehat{F}_{j+\frac{1}{2}}$ are obtained by the standard WENO reconstruction on $F(U)$. In addition, the source term approximation reduces to

$$
\left(\frac{K}{3 \rho \sqrt{\pi}} \frac{A}{A_{0}}\left(A_{0}^{\frac{3}{2}}\right)_{x}\right)_{j}=\left(\frac{K}{3 \rho \sqrt{\pi}}\left(A_{0}^{\frac{3}{2}}\right)_{x}\right)_{j}
$$

which is computed by the WENO reconstruction. Therefore, the flux and source term approximation reduce to the same WENO reconstruction for $\left(\frac{K}{3 \rho \sqrt{\pi}}\left(A^{\frac{3}{2}}\right)_{x}\right)_{j}$ due to $A=A_{0}$, and cancel with each other, which leads to the desired well-balanced property.

We now summarize the complete procedure of the high order well-balanced finite difference WENO-LF scheme with the local characteristic decomposition for solving the blood flow model (1.1):

Step 1 Reformulate the source term and rewrite the governing equations in the form (3.1).

Step 2 Approximate the derivative term in the source term of momentum equation in (3.1) by splitting them as in (3.6) and then applying the standard WENO procedure with flux splitting on them to obtain $\left(\widehat{A_{0}^{\frac{3}{2}}}\right)_{j+\frac{1}{2}}$ as in (3.7).

Step 3 At each time step, perform the usual WENO-LF approximation on the flux derivative $F(U)_{x}$ with a modified flux splitting.

Step 4 Evaluate the source term at the current time step by (3.3) using the derivative term approximation from Step 2. 
Step 5 Add up the residues of the numerical flux and source term approximations, and forward in time by Runge-Kutta methods.

\section{Numerical results}

In this section, we carry out extensive numerical experiments to demonstrate the performance of the proposed well-balanced WENO scheme. In all the computations, we apply the third order TVD Runge-Kutta method (2.7). The CFL number is taken as 0.6 for stability criteria.

\subsection{To test the order of accuracy}

We apply this example to test the order of accuracy of the resulting method. We take the following initial conditions:

$$
A(x, 0)=\sin ^{2}(\pi x), \quad Q(x, 0)=\sin (\pi x)+\cos (\pi x) \quad \text { and } \quad A_{0}(x)=\cos ^{2}(\pi x),
$$

on a computational domain $[0,2]$ based on the following parameters: $K=1 \times 10^{8} \mathrm{~Pa} / \mathrm{m}$, $\rho=1060 \mathrm{~kg} / \mathrm{m}^{3}$.

We impose this problem with periodic boundary conditions at the two endpoints. Then, we solve this example up to $t=0.01 \mathrm{~s}$ and get reference solutions on a mesh with 2000 cells. We present the errors and the order of accuracy in Table 1. It is clear that the current method obtains the expected fifth order of accuracy.

Table 1: $L^{1}$ errors and numerical orders of accuracy for the example of Section 4.1.

\begin{tabular}{||c|cc|cc||}
\hline \multirow{2}{*}{$N$} & \multicolumn{2}{|c|}{$A$} & \multicolumn{2}{c||}{$Q$} \\
\cline { 2 - 5 } & $L^{1}$ error & Order & $L^{1}$ error & Order \\
\hline 25 & $1.75 \mathrm{E}-02$ & & $1.07 \mathrm{E}-02$ & \\
50 & $2.17 \mathrm{E}-03$ & 3.01 & $1.94 \mathrm{E}-03$ & 2.46 \\
100 & $3.25 \mathrm{E}-04$ & 2.74 & $2.77 \mathrm{E}-04$ & 2.81 \\
200 & $2.32 \mathrm{E}-05$ & 3.81 & $1.99 \mathrm{E}-05$ & 3.80 \\
400 & $9.30 \mathrm{E}-07$ & 4.64 & $9.56 \mathrm{E}-07$ & 4.38 \\
800 & $3.09 \mathrm{E}-08$ & 4.91 & $3.25 \mathrm{E}-08$ & 4.88 \\
1600 & $9.60 \mathrm{E}-10$ & 5.01 & $9.94 \mathrm{E}-10$ & 5.03 \\
\hline
\end{tabular}

\subsection{The ideal tourniquet}

This example is similar with the dam break problem in shallow water equations, namely Stoker's solution [23]. Here, we consider the analogue of this problem in blood flow model: a tourniquet is applied and we remove it instantaneously. We consider the following 


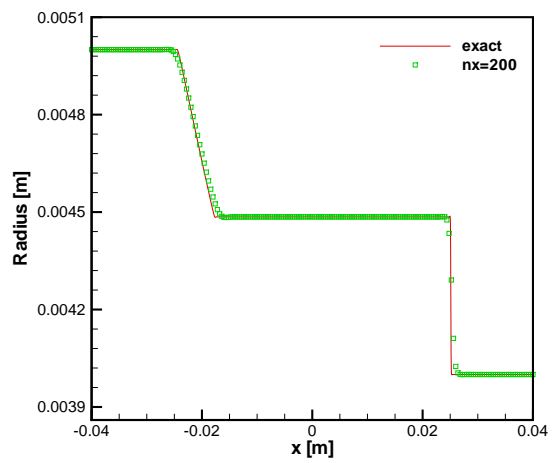

(a)

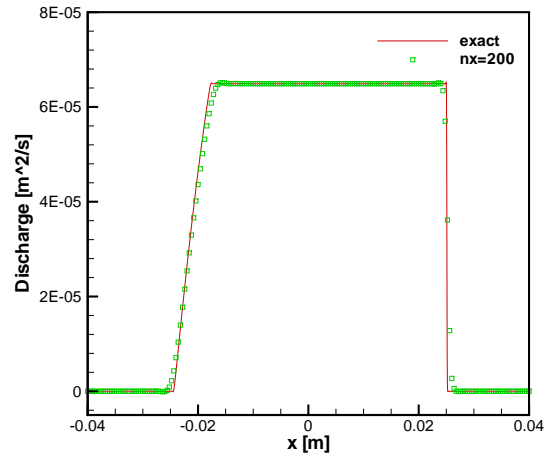

(b)

Figure 1: The numerical solutions of the ideal tourniquet problem in Section 4.2 on a mesh with 200 cells at $t=0.005$ s. Radius (a) and discharge (b).

initial conditions

$$
A(x, 0)=\left\{\begin{array}{ll}
\pi R_{L}^{2}, & \text { if } x \leq 0, \\
\pi R_{R}^{2}, & \text { otherwise, }
\end{array} \text { and } Q(x, 0)=0,\right.
$$

on a computational domain $[-0.04,0.04]$ based on the following parameters: $K=1 \times$ $10^{7} \mathrm{~Pa} / \mathrm{m}, \rho=1060 \mathrm{~kg} / \mathrm{m}^{3}, R_{L}=5 \times 10^{-3} \mathrm{~m}, R_{R}=4 \times 10^{-3} \mathrm{~m}$ and $R_{0}=3 \times 10^{-3} \mathrm{~m}$.

We impose this problem with transmissive boundary conditions. Then, we solve this example on the mesh with 200 cells up to $t=0.005$ s and present the numerical solutions against the exact ones in Fig. 1. It is clear that the numerical results fit well with the exact ones and keep steep shock transitions.

\subsection{Wave equation}

The following quasi-stationary test case presented in [12] is chosen to demonstrate the capability of the proposed scheme to compute the spreading of a small perturbation of a steady state. In this case, we recover the behavior of the wave equation.

Herein, we apply the following initial data:

$A(x, 0)=\left\{\begin{array}{ll}\pi R_{0}^{2}, & \text { if } x \in\left[0, \frac{40 L}{100}\right] \cup\left[\frac{60 L}{100}, L\right], \\ \pi R_{0}^{2}\left[1+\epsilon \sin \left(\pi \frac{x-40 L / 100}{20 L / 100}\right)\right]^{2}, & \text { if } x \in\left[\frac{40 L}{100}, \frac{60 L}{100}\right],\end{array} \quad Q(x, 0)=0\right.$,

on a computational domain $[0,0.16]$ coupled with transmissive boundary conditions. The following parameters have been used for this example: $\epsilon=5 \times 10^{-3}, K=10^{8} \mathrm{~Pa} / \mathrm{m}, \rho=$ $1060 \mathrm{~kg} / \mathrm{m}^{3}, R_{0}=4 \times 10^{-3} \mathrm{~m}$ and $L=0.16 \mathrm{~m}$. 


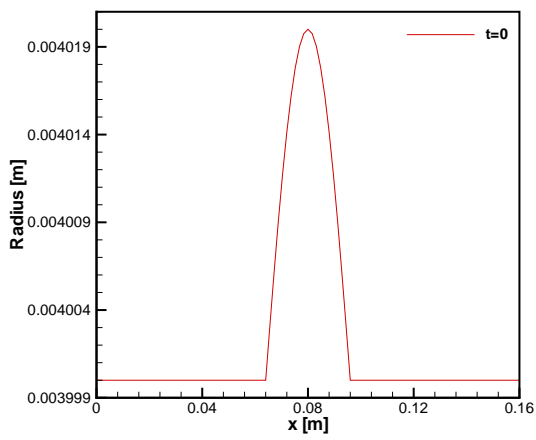

Figure 2: The initial radius of the wave equation in Section 4.3.

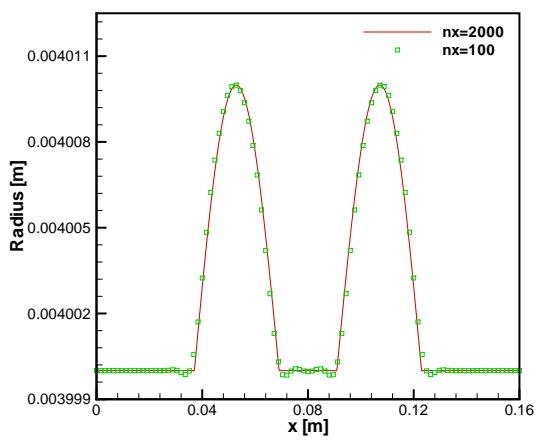

(a)

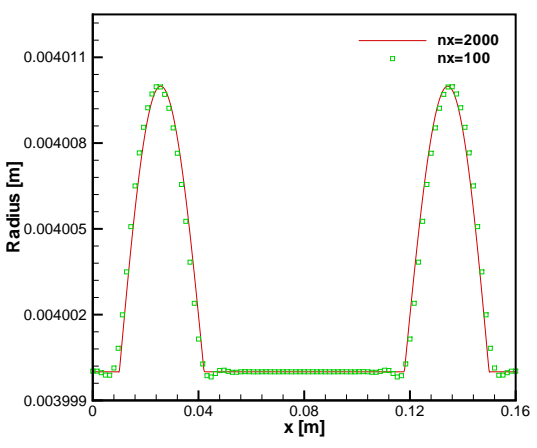

(b)

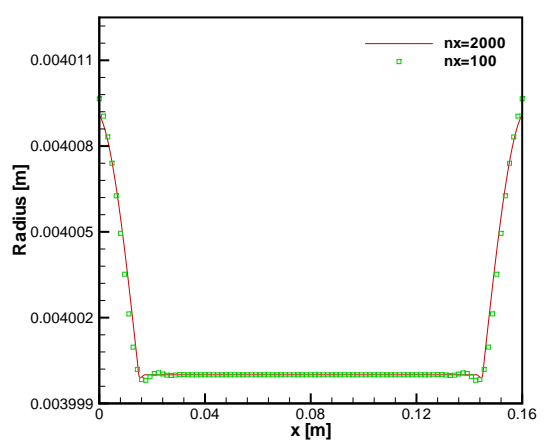

(c)

Figure 3: The numerical solutions of the wave equation problem in Section 4.3 on a mesh with 100 cells. Radius at $t=0.002 \mathrm{~s}(\mathrm{a}), t=0.004 \mathrm{~s}(\mathrm{~b})$, and $t=0.006 \mathrm{~s}$ (c), respectively.

The initial radius is presented in Fig. 2. We show the numerical solutions on a mesh with 200 cells at $t=0.002 \mathrm{~s}, 0.004 \mathrm{~s}$, and $0.006 \mathrm{~s}$ against the reference solutions, respectively 
in Fig. 3. These figures strongly suggest that the numerical solutions agree well with the reference ones.

\subsection{The man at eternal rest}

The purpose of this example is to verify that the current WENO scheme indeed maintains the well-balanced property.

Herein, we consider a configuration with no flow and with a change of radius $R_{0}(x)$, this is the case for a dead man with an aneurism. Thus, for the initial conditions, the section of the artery is not constant with the following form

$$
R(x, 0)=R_{0}(x)= \begin{cases}\widetilde{R}, & \text { if } x \in\left[0, x_{1}\right] \cup\left[x_{4}, L\right], \\ \widetilde{R}+\frac{\Delta R}{2}\left[\sin \left(\frac{x-x_{1}}{x_{2}-x_{1}} \pi-\frac{\pi}{2}\right)+1\right], & \text { if } x \in\left[x_{1}, x_{2}\right], \\ \widetilde{R}+\Delta R, & \text { if } x \in\left[x_{2}, x_{3}\right], \\ \widetilde{R}+\frac{\Delta R}{2}\left[\cos \left(\frac{x-x_{3}}{x_{4}-x_{3}} \pi\right)+1\right], & \text { if } x \in\left[x_{3}, x_{4}\right],\end{cases}
$$

on the computational domain $[0,0.14]$ with $\widetilde{R}=4 \times 10^{-3} \mathrm{~m}, \Delta R=10^{-3} \mathrm{~m}, K=10^{8} \mathrm{~Pa} / \mathrm{m}$, $\rho=1060 \mathrm{~kg} / \mathrm{m}^{3}, L=0.14 \mathrm{~m}, x_{1}=10^{-2} \mathrm{~m}, x_{2}=3.05 \times 10^{-2} \mathrm{~m}, x_{3}=4.95 \times 10^{-2} \mathrm{~m}$ and $x_{4}=7 \times$ $10^{-2} \mathrm{~m}$. In addition, the initial velocity is zero. We impose this problem with transmissive boundary conditions and compute this example up to $t=5 \mathrm{~s}$.

In order to show that the well-balanced property is maintained up to machine round off error, tests are run using single, double and quadruple precisions, respectively. The $L^{1}$ and $L^{\infty}$ errors calculated for $A$, and $Q$ are presented in Table 2 . It can be clearly seen that the $L^{1}$ and $L^{\infty}$ errors are all at the level of round off errors associated with different precisions, which verify that the current WENO scheme indeed maintains the steady state $(u=0)$ and thus its well-balanced property accordingly.

In Fig. 4, we present the radius at $t=5 \mathrm{~s}$ on a mesh with 200 cells against a reference solution obtained with a much refined 2000 cells. In addition, we run the same numerical test using the non-well-balanced WENO scheme, with a straightforward integration of the source term, and show their result in Fig. 4 for comparison. It is obvious that the result of well-balanced WENO scheme is in good agreement with the reference solution for the case, while non-well-balanced WENO scheme produces spurious oscillations.

Table 2: $L^{1}$ and $L^{\infty}$ error for different precisions for the man at eternal rest.

\begin{tabular}{||c|cc|cc||}
\hline \multirow{2}{*}{ Precision } & \multicolumn{2}{|c|}{$L^{1}$ error } & \multicolumn{2}{c||}{$L^{\infty}$ error } \\
\cline { 2 - 5 } & $A$ & $Q$ & $A$ & $Q$ \\
\hline Single & $1.14 \mathrm{E}-07$ & $1.46 \mathrm{E}-07$ & $4.98 \mathrm{E}-07$ & $5.32 \mathrm{E}-07$ \\
\hline Double & $3.82 \mathrm{E}-16$ & $2.82 \mathrm{E}-16$ & $2.31 \mathrm{E}-16$ & $2.45 \mathrm{E}-15$ \\
\hline Quadruple & $2.91 \mathrm{E}-31$ & $5.69 \mathrm{E}-32$ & $6.50 \mathrm{E}-33$ & $8.52 \mathrm{E}-32$ \\
\hline
\end{tabular}




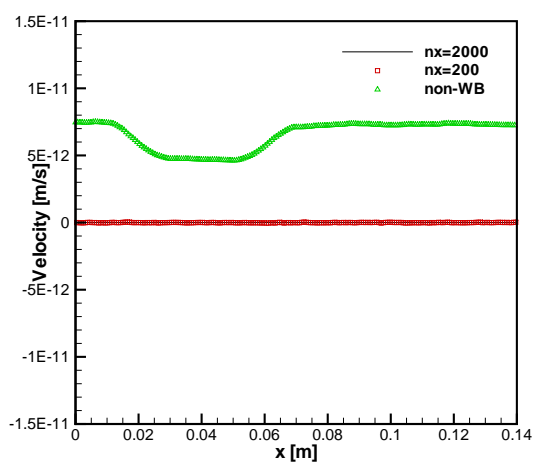

Figure 4: The man at eternal rest problem in Section 4.4 at $t=5 \mathrm{~s}$. The result of the well-balanced scheme with 200 and 2000 cells, and that of the non-well-balanced (denoted by non-WB) scheme with 200 cells.

\subsection{Propagation of a pulse to an expansion}

In this section, we test the case of a pulse in a section $R_{R}$ passing trough an expansion: $A_{L}>A_{R}$, taking the following parameters: $K=1.0 \times 10^{8} \mathrm{~Pa} / \mathrm{m}, L=0.16 \mathrm{~m}, \rho=1060 \mathrm{~kg} / \mathrm{m}^{3}$, $R_{L}=5 \times 10^{-3} \mathrm{~m}, R_{R}=4 \times 10^{-3} \mathrm{~m}, \Delta R=1.0 \times 10^{-3} \mathrm{~m}$. We take a decreasing shape on a rather small scale:

$$
R_{0}(x)= \begin{cases}R_{R}+\Delta R, & \text { if } x \in\left[0, x_{1}\right], \\ R_{R}+\frac{\Delta R}{2}\left[1+\cos \left(\frac{x-x_{1}}{x_{2}-x_{1}} \pi\right)\right], & \text { if } x \in\left[x_{1}, x_{2}\right], \\ R_{R}, & \text { otherwise }\end{cases}
$$

with $x_{1}=\frac{19 L}{40}, x_{2}=\frac{L}{2}$. As initial conditions, we consider a fluid at rest $\left(Q(x, 0)=0 \mathrm{~m}^{3} / \mathrm{s}\right)$ and the following perturbation of radius:

$$
R(x, 0)= \begin{cases}R_{0}(x)\left[1+\epsilon \sin \left(\frac{100}{20 L} \pi\left(x-\frac{65 L}{100}\right)\right)\right], & \text { if } x \in\left[\frac{65 L}{100}, \frac{85 L}{100}\right], \\ R_{0}(x), & \text { otherwise, }\end{cases}
$$

with $\epsilon=5.0 \times 10^{-3}$ and with transmissive boundary conditions.

In Fig. 5, we present the numerical results against the reference solutions at $t=0.002 \mathrm{~s}$ and $t=0.006 \mathrm{~s}$. At first, we get two travelling waves, one spreading to the left and the other one going to the right, as illustrated on Fig. 5 at time $t=0.002 \mathrm{~s}$.

The numerical solutions are in good agreement with the reference ones and are comparable with those in [12]. 


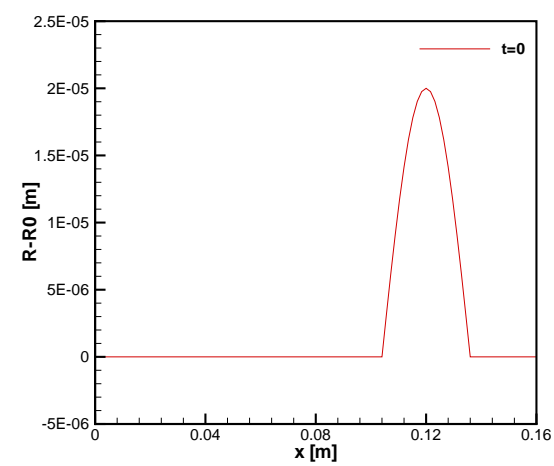

(a)

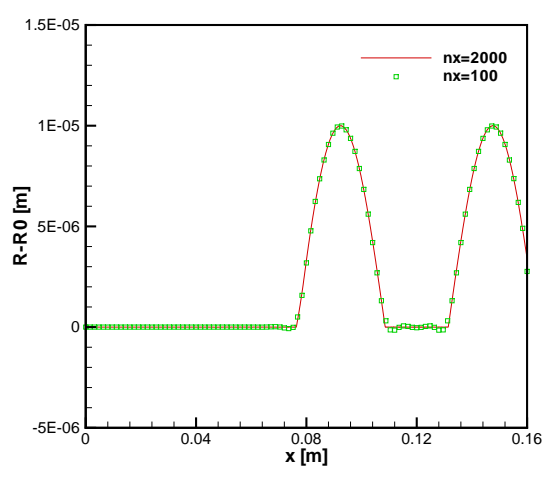

(b)

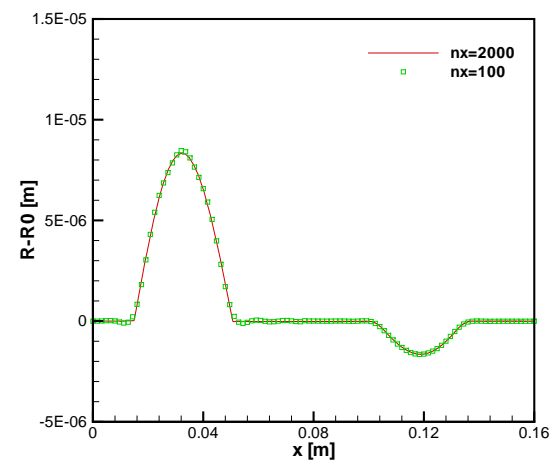

(c)

Figure 5: The numerical solutions of the propagation of a pulse to an expansion in Section 4.5 on a mesh with 100 cells. The errors $R-R_{0}$ at $t=0 \mathrm{~s}(\mathrm{a}), t=0.002 \mathrm{~s}(\mathrm{~b})$ and $t=0.006 \mathrm{~s}(\mathrm{c})$.

\subsection{Propagation of a pulse from an expansion}

Then, we consider a pulse propagating from an expansion. So, the parameters are the same as in the Section 4.5, only the initial radius is changed:

$$
R(x, 0)= \begin{cases}R_{0}(x)\left[1+\epsilon \sin \left(\frac{100}{20 L} \pi\left(x-\frac{15 L}{100}\right)\right)\right], & \text { if } x \in\left[\frac{15 L}{100}, \frac{35 L}{100}\right], \\ R_{0}(x), & \text { otherwise, }\end{cases}
$$

with $\epsilon=5.0 \times 10^{-3}$. Similarly, we also impose this problem with transmissive boundary conditions.

In Fig. 6, we demonstrate the numerical results against the reference solutions at $t=$ $0.002 \mathrm{~s}$ and $t=0.006 \mathrm{~s}$. Similarly, the numerical solutions fit well with the reference ones and are comparable with those in [12]. 


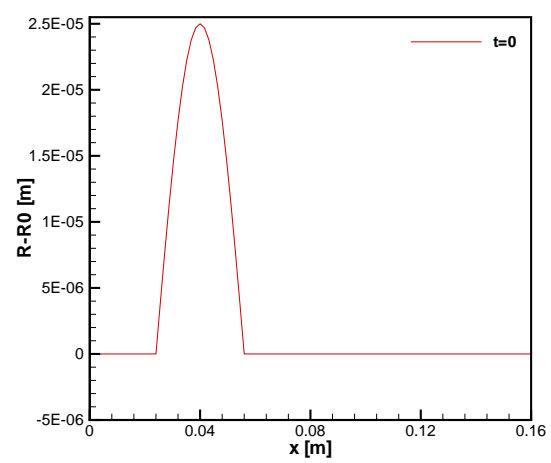

(a)

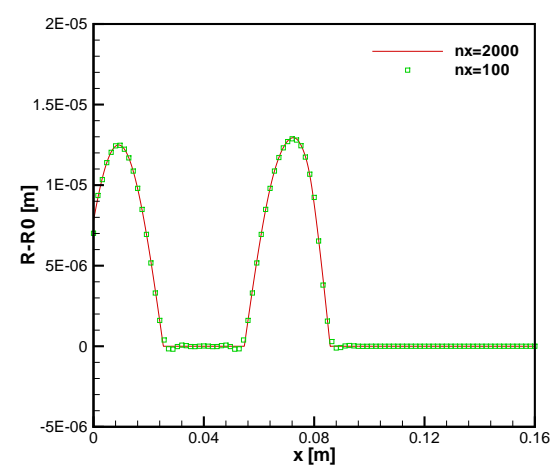

(b)

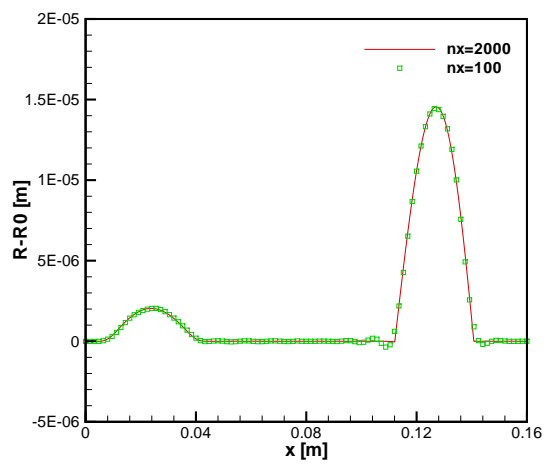

(c)

Figure 6: The numerical solutions of the propagation of a pulse from an expansion in Section 4.6 on a mesh with 100 cells. The errors $R-R_{0}$ at $t=0 \mathrm{~s}(\mathrm{a}), t=0.002 \mathrm{~s}$ (b) and $t=0.006 \mathrm{~s}$ (c).

\subsection{Wave damping}

In this last test case, we look at the viscous damping term in the linearized momentum equation. This is an analogue of the Womersley problem [24], we consider a periodic signal at the inflow with a constant section at rest. We consider the following model coupled with the linear friction term

$$
\left\{\begin{array}{l}
A_{t}+Q_{x}=0, \\
Q_{t}+\left(\frac{Q^{2}}{A}+\frac{K}{3 \rho \sqrt{\pi}} A^{\frac{3}{2}}\right)_{x}=\frac{K A}{2 \rho \sqrt{\pi} \sqrt{A_{0}}}\left(A_{0}\right)_{x}-C_{f} \frac{Q}{A},
\end{array}\right.
$$

where $C_{f}=8 \pi v$ with $v$ being the blood viscosity. For the friction term $-C_{f} \frac{Q}{A}$, we directly use its grid point value. We consider this example on the computational domain $[0,3]$ 
subject to the given initial conditions

$$
\left\{\begin{array}{l}
A(x, 0)=\pi R_{0}^{2} \\
Q(x, 0)=0
\end{array}\right.
$$

companied by the following parameters: $K=1 \times 10^{8} \mathrm{~Pa} / \mathrm{m}, \rho=1060 \mathrm{~kg} / \mathrm{m}^{3}, R_{0}=4 \times 10^{-3} \mathrm{~m}$. We solve this example up to $t=25 \mathrm{~s}$.

Subsequently, we obtain a damping wave [12]

$$
Q(t, x)= \begin{cases}0, & \text { if } k_{r} x>\omega t \\ Q_{\mathrm{amp}} \sin \left(\omega t-k_{r} x\right) e^{k_{i} x}, & \text { if } k_{r} x \leq \omega t\end{cases}
$$

with

$$
\begin{aligned}
& k_{r}=\left[\frac{\omega^{4}}{c_{0}^{4}}+\left(\frac{\omega C_{f}}{\pi R_{0}^{2} c_{0}^{2}}\right)^{2}\right]^{\frac{1}{4}} \cos \left(\frac{1}{2} \arctan \left(-\frac{C_{f}}{\pi R_{0}^{2} \omega}\right)\right), \\
& k_{i}=\left[\frac{\omega^{4}}{c_{0}^{4}}+\left(\frac{\omega C_{f}}{\pi R_{0}^{2} c_{0}^{2}}\right)^{2}\right]^{\frac{1}{4}} \sin \left(\frac{1}{2} \arctan \left(-\frac{C_{f}}{\pi R_{0}^{2} \omega}\right)\right), \\
& w=2 \pi / T_{\text {pulse }}=2 \pi /(0.5 \mathrm{~s}), \\
& c_{0}=\sqrt{\frac{k \sqrt{A_{0}}}{2 \rho \sqrt{\pi}}}=\sqrt{\frac{k R_{0}}{2 \rho}} .
\end{aligned}
$$

For the treatment of the boundary conditions, we impose the incoming discharge

$$
Q_{b}(t)=Q_{\mathrm{amp}} \sin (w t) \mathrm{m}^{3} / \mathrm{s},
$$

at $x=0 \mathrm{~m}$ with $Q_{\mathrm{amp}}=3.45 \times 10^{-7} \mathrm{~m}^{3} / \mathrm{s}$ being the amplitude of the inflow discharge. As the flow is subcritical, the discharge is imposed at the outflow boundary, thanks to (4.2) at $x=3 \mathrm{~m}$.

In Fig. 7, we present the numerical results against the exact solutions at $t=25 \mathrm{~s}$ with different $C_{f}$. It is obvious that the numerical solutions are in good agreement with the exact solutions and are comparable with those in [12].

\section{Concluding remarks}

In this paper, we present a well-balanced finite difference WENO scheme to solve the blood flow model based on the reformulation of the equation and a novel source term. The current scheme maintains the well-balanced property for steady state, and at the same time keeps its original high order accuracy. Extensive numerical examples are carried out to demonstrate the well-balanced property, high order accuracy, and steep shock transitions of the proposed methods. The research on the finite volume WENO schemes are ongoing. 


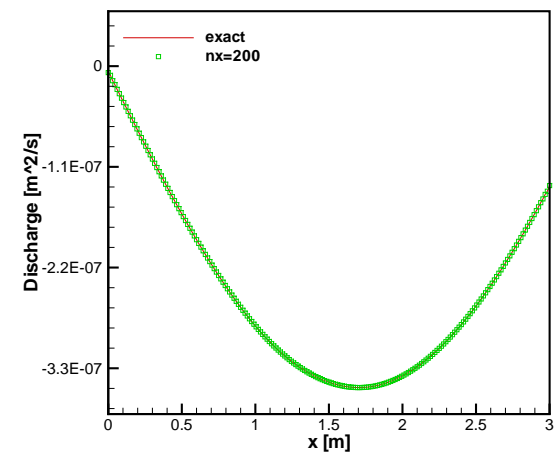

(a)

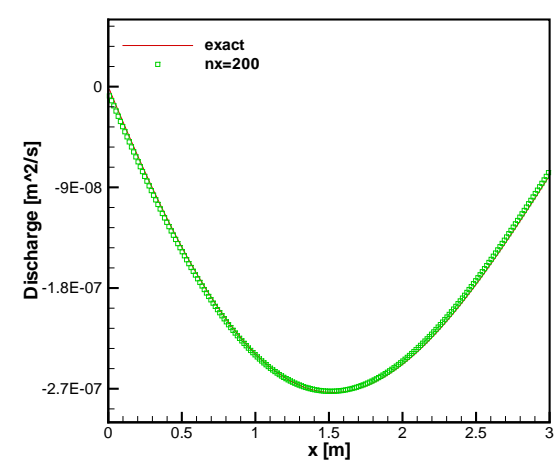

(c)

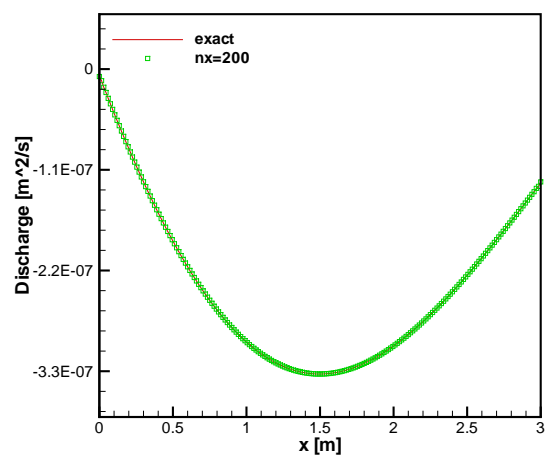

(b)

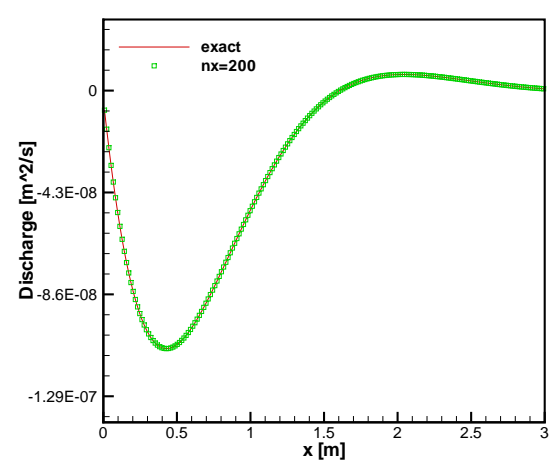

(d)

Figure 7: The numerical solutions of the propagation of a pulse to and from an expansion in Section 4.7 on a mesh with 200 cells at $t=25 \mathrm{~s}$. The damping of a discharge wave with $C_{f}=0$ (a), $C_{f}=0.000022$ (b), $C_{f}=0.000202(\mathrm{c})$ and $C_{f}=0.005053(\mathrm{~d})$.

\section{Acknowledgements}

The authors would like to thank the support of the Natural Science Foundation of China through Grants Nos. 11201254 and 41476101, the Natural Science Foundation of Shandong Province of China through Grants Nos. ZR2014DM017 and ZR2015PF002, and the Project for Scientific Plan of Higher Education in Shandong Province of China through Grant No. J12LI08.

\section{References}

[1] L. Formaggia, D. Lamponi, M. Tuveri And A. Veneziani, Numerical modeling of 1D arterial networks coupled with a lumped parameters description of the heart, Comput. Meth. Biomech. Biomed. Eng., 9 (2006), pp. 273-288. 
[2] V. B. Kolachalama, N. W. Bressloff, P. B. Nair and C. P. Shearman, Predictive Haemodynamics in a one-dimensional human carotid artery bifurcation, Part I: application to stent design, IEEE T Bio-Med. Eng., 54 (2007), pp. 802-812.

[3] D. XIU AND S. J. SHERWIN, Parametric uncertainty analysis of pulse wave propagation in a model of a human arterial network, J. Comput. Phys., 226 (2007), pp. 1385-1407.

[4] J. M. GREENBERG AND A. Y. LeRouX, A well-balanced scheme for the numerical processing of source terms in hyperbolic equations, SIAM J. Num. Anal., 33 (1996), pp. 1-16.

[5] Y. L. XING, C. W. SHU AND S. NOELLE, On the advantage of well-balanced schemes for movingwater equilibria of the shallow water equations, J. Sci. Comput., 48 (2011), pp. 339-349.

[6] S. Noelle, Y. L. XING AND C. W. SHU, High-order well-balanced schemes, in: Numerical Methods for Balance Laws (G. Puppo and G. Russo eds), Quaderni di Matematica, (2010).

[7] R. J. LEVEQUE, Balancing source terms and flux gradients on high-resolution Godunov methods: the quasi-steady wave-propagation algorithm, J. Comput. Phys., 146 (1998), pp. 346-3658.

[8] B. Perthame And C. SimeOni, A kinetic scheme for the Saint-Venant system with a source term, Calcolo, 38 (2001), pp. 201-231.

[9] K. XU, A well-balanced gas-kinetic scheme for the shallow-water equations with source terms, J. Comput. Phys., 178 (2002), pp. 533-562.

[10] Y. L. XING AND C. W. SHU, High order finite difference WENO schemes with the exact conservation property for the shallow water equations, J. Comput. Phys., 208 (2005), pp. 206-227.

[11] Y. L. XING AND C. W. SHU, A survey of high order schemes for the shallow water equations, J. Math. Stud., 47 (2014), pp. 221-2494.

[12] O. DeLESTRE AND P. Y. LAGRÉE, A well-balanced finite volume scheme for blood flow simulation, Int. J. Numer. Meth. F1., 72 (2013), pp. 177-205.

[13] M. Wibmer, One-Dimensional Simulation of Arterial Blood flow with Applications, PhD Thesis, eingereicht an der Technischen Universitat Wien, Fakultat fur Technische Naturwissenschaften und Informatik, January, (2004).

[14] N. Cavallini, V. CAlefFi AND V. COSCiA, Finite volume and WENO scheme in onedimensional vascular system modelling, Comput. Math. Appl., 56 (2008), pp. 2382-2397.

[15] N. CAVAllini AND V. Coscia, One-dimensional modelling of venous pathologies: finite volume and WENO schemes, in Advances in Mathematical Fluid Mechanics, R. Rannacher, A. Sequeira (eds), Springer, Berlin Heidelberg, (2010).

[16] L. O. MÜLlER, C. PARÉS AND E. F. TORO, Well-balanced high-order numerical schemes for onedimensional blood flow in vessels with varying mechanical properties, J. Comput. Phys., 242 (2013), pp. 53-85.

[17] J. MuRILlo AND P. GarCíA-NAVARRO, A Roe type energy balanced solver for 1D arterial blood flow and transport, Comput. Fluids, 117 (2015), pp. 149-167.

[18] Z. Z. WANG, G. Li AND O. DELESTRE, Well-balanced finite difference weighted essentially nonoscillatory schemes for the blood flow model, Int. J. Numer. Meth. Fl., 82 (2016), pp. 607-622.

[19] G. JiANG AND C. W. SHU, Efficient implementation of weighted ENO schemes, J. Comput. Phys., 126 (1996), pp. 202-228.

[20] C. W. SHU, Essentially non-oscillatory and weighted essentially non-oscillatory schemes for hyperbolic conservation laws, NASA/CR-97-206253, ICASE Report NO. 97-65.

[21] C. W. SHU, High order weighted essentially nonoscillatory schemes for convection dominated problems, SIAM Rev., 51 (2009), pp. 82-126.

[22] C. W. SHU AND S. OSHER, Efficient implementation of essentially non-oscillatory shock-capturing schemes, J. Comput. Phys., 77 (1988), pp. 439-471.

[23] O. Delestre, C. Lucas, P. A. Ksinant, F. Darboux, C. Laguerre, T. N. T. Vo, F. James 
AND S. CORDIER, SWASHES: a complilation of shallow water analytic solutions for hydraulic and environmental studies, Int. J. Numer. Meth. Fl., 72 (2013), pp. 269-300.

[24] J. Womersley, On the oscillatory motion of a viscous liquid in thin-walled elastic tube, I. Phil. Mag., 46 (1955), pp. 199-221. 